\title{
A New Look at Magnetic White Dwarfs
}

\author{
F. Hardy, ${ }^{1}$ P. Dufour, ${ }^{1}$ and S. Jordan ${ }^{2}$ \\ ${ }^{1}$ Université de Montréal, Montréal, QC, Canada; hardy@astro.umontreal.ca \\ ${ }^{2}$ Astronomisches Rechen-Institut am Zentrum für Astronomie der Universität Heidelberg, Germany; jordan@ari.uni-heidelberg.de
}

\section{Abstract}

We present a homogeneous photometric analysis of a large sample of magnetic DA white dwarfs as well as selected results from a spectrophotometric analysis of magnetic carbon-dominated atmosphere white dwarfs. We find the presence of oxygen absorption lines in 3 of the 5 objects.

\section{Introduction}

Atmospheric parameters for magnetic white dwarfs are notoriously difficult to obtain due to the complex line profiles and positions that arise as a result of a possibly variable magnetic strength (and corresponding line splitting) across the surface of the star. Moreover, the reliability of the mass determination from spectral line fitting is somewhat questionable since it is still unclear how to treat Stark and Zeeman broadening simultaneously when the fields are strong. Nevertheless, decent estimations can be obtained from fits to the energy distribution when parallax measurement are available, a situation that was unfortunately possible only for about two dozen objects before the Gaia mission. Here we present a homogenous analysis of all known magnetic DAs with magnetic field greater than 0.5 MG based on Gaia parallax measurement (Gaia Collaboration et al., 2016, 2018) and Pan-STARRS photometry (Chambers et al. 2016). We also revisit spectroscopic observations of magnetic carbon atmosphere white dwarfs using grids of magnetic synthetic spectra calculated for various dipole geometry in the Paschen-Back regime, extending the analysis of Dufour et al. (2015) where only centered dipole with $45^{\circ}$ inclination were explored.

\section{Methodology}

We first fit the energy distribution with grids of nonmagnetic synthetic spectra (hydrogen- or carbon-rich atmospheres) to obtain the effective temperature and solid angle $\pi(R / D)^{2}$ of our objects. Since the distance $D$ is known from Gaia trigonometric parallax measurements, the radius $R$ of each star can be determined and converted to mass using evolutionary models. Although strong magnetic field may push some lines far from their zero field position and thus affect the flux of the star in some photometric bands, this represents a reasonable first approximation for most magnetic field strengths encountered (a full self-consistent analysis will be reported elsewhere).

\section{Results}

\subsection{Mass distribution of magnetic DA stars}

Figure 1 shows the mass distribution for our sample of 138 DA magnetic white dwarfs with available Gaia trigonometric parallax measurements and Pan-STARRS photometry. We find that the mass distribution of magnetic white dwarfs significantly differs from that of non-magnetic DA white dwarfs. First, masses are more or less evenly distributed between 0.5 and 1.2 solar mass with no significant peak near $0.6 M_{\odot}$. There is also a significant fraction of the sample with masses above $0.8 M_{\odot}$. We find that those massive white dwarfs, contrary to the DQ white dwarfs (see proceedings contribution by Simon Coutu) show a tangential velocity distribution that is consistent with that expected from a young population, indicating that these objects are probably not the result of merging white dwarfs. Note that a self-consistent atmospheric determination using grids of magnetic synthetic spectra, which will be presented in due time, may affect the parameters determination of the most highly magnetic objects (but the overall shape of this distribution is not likely to change very much since only $13 \%$ of magnetic DAs shown here have fields above $50 \mathrm{MG}$ ).

\subsection{Magnetic carbon atmosphere white dwarfs}

In the presence of a strong magnetic field, it is often difficult to identify elements since lines have been split and shifted to places where they are not easily recognized (Dufour et al., 2008, 2013). Although oxygen, in addition to carbon, was suspected to be present in some 

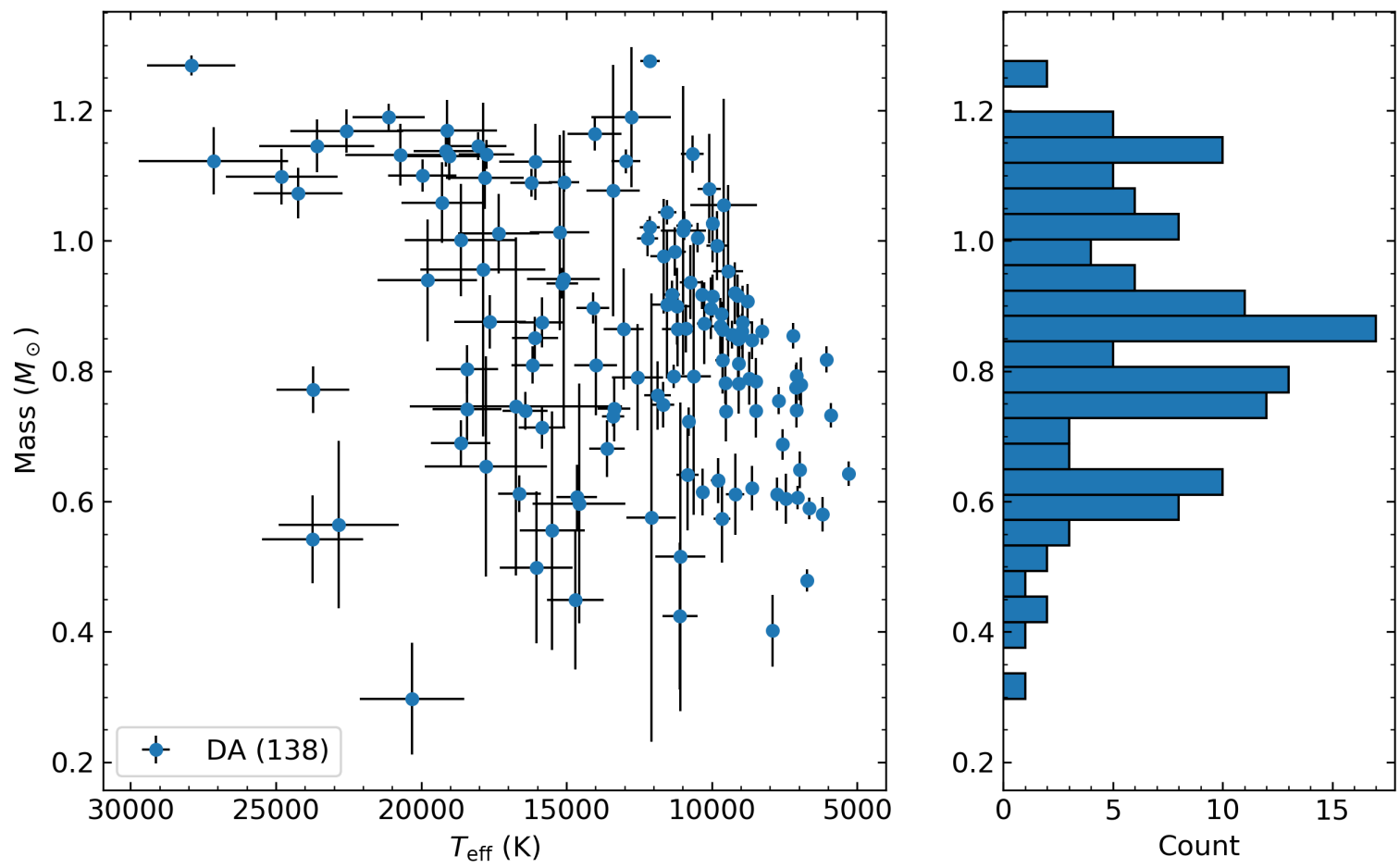

Figure 1: Masses of magnetic white dwarfs with magnetic fields greater than 0.5 MG as a function of effective temperature. Right plot shows the corresponding mass histogram.

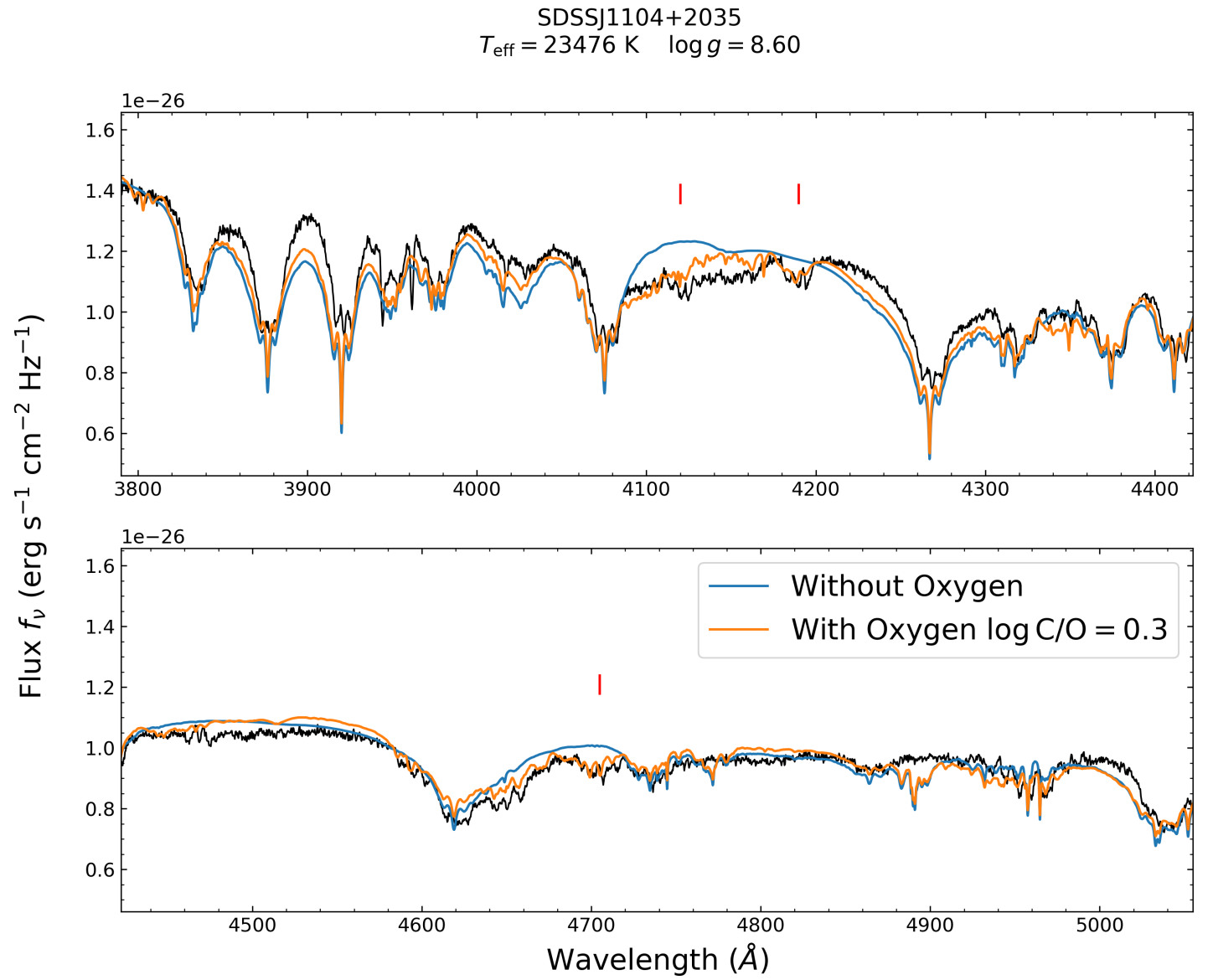

Figure 2: Synthetic spectrum with pure carbon composition (blue) and with oxygen (orange) for the star SDSSJ1104+2035. The red ticks show the locations of the identified oxygen features. 
Table 1: Mean of the magnetic field geometry parameters for the 5 Hot DQ stars presented in Figures 2 and 3 The Regions used column is the number of wavelength ranges used in the fitting process. The numbers in parentheses are the standard deviations.

\begin{tabular}{cclcc} 
Name (SDSS) & Regions used & \multicolumn{1}{c}{$B(\mathrm{MG})$} & $i$ (degrees) & $a_{z}\left(R_{*}\right)$ \\
\hline J0005-1002 & 10 & $4.2(1.0)$ & $60(15)$ & $0.11(0.15)$ \\
J1104+2035 & 11 & $1.22(0.21)$ & $67(18)$ & $0.39(0.16)$ \\
J1337-0026 & 11 & $0.73(0.20)$ & $58(17)$ & $0.11(0.15)$ \\
J1402+3818 & 4 & $3.41(0.10)$ & $60(19)$ & $0.02(0.26)$ \\
J2200-0741 & 14 & $2.46(0.40)$ & $50(19)$ & $0.08(0.15)$
\end{tabular}

objects, no detailed modeling has yet been done to confirm these suspicions. Here we compute grids of magnetic synthetic spectra for pure carbon atmospheres, as well as atmospheres containing traces of oxygen, hydrogen and helium, in order to formally identify these elements in our spectra if present. Our grids cover a wide range of magnetic field intensity $B$, inclination $i$ and $z$-axis offsets $a_{z}$ assuming a dipolar geometry and newly determined photometric atmospheric parameters based on Gaia parallax measurements for each object. The splitting of spectral lines of carbon and oxygen are treated in the Paschen-Back regime following the prescription described in detail in Hardy et al. (in preparation, see also Dufour et al., 2015). The characteristics of the magnetic field (strength, inclination and offset) are then obtained using an evolutionary algorithm to explore the whole parameter space (and thus avoid local minima solutions) since gradient-based methods are ill-suited in cases where many extrema are present. We find that the fitting algorithm works best by focusing on small wavelength range (regions) containing only a few lines. We thus find the global solution by calculating the mean of the parameters obtained in all the spectral regions considered (see Table 1).

\section{Conclusions}

We obtained the first homogeneous magnetic DA mass distribution for a sample of 138 known white dwarfs with magnetic fields larger than 0.5 MG. The distribution is significantly different from that of non-magnetic DAs. We also find that 3 of the 5 Hot DQ white dwarfs studied show traces of oxygen, and one could in fact be helium-rich. Future work will determine the atmospheric parameters in a self-consistent way (i.e., stellar structure calculated under the same assumption as the synthetic spectra with magnetic field).

\section{References}

Chambers K. C., et al., 2016, preprint, (arXiv:1612.05560)

Dufour P., Fontaine G., Liebert J., Schmidt G. D., Behara N., 2008, ApJ, 683, 978

Dufour P., Vornanen T., Bergeron P., Fontaine Berdyugin A., Krzesiński J., Stachowski G., Moskalik P., Bajan K., 2013, in 18th European White Dwarf Workshop.. p. 167

Dufour P., et al., 2015, in 19th European Workshop on White Dwarfs. p. 37

Gaia Collaboration et al., 2016, A\&A, 595, A1

Gaia Collaboration et al., 2018, A\&A, 616, A1 

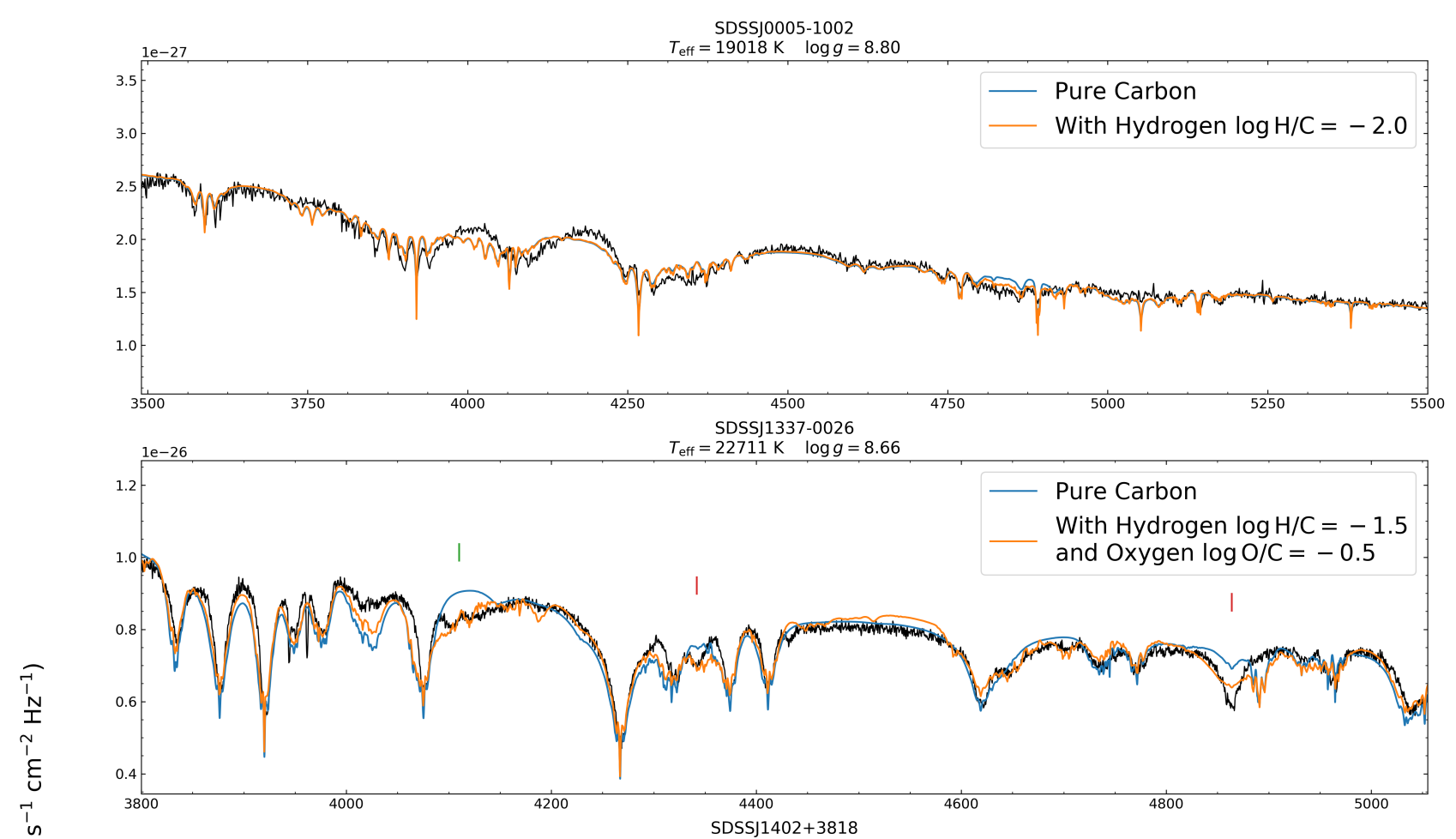

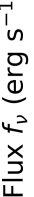
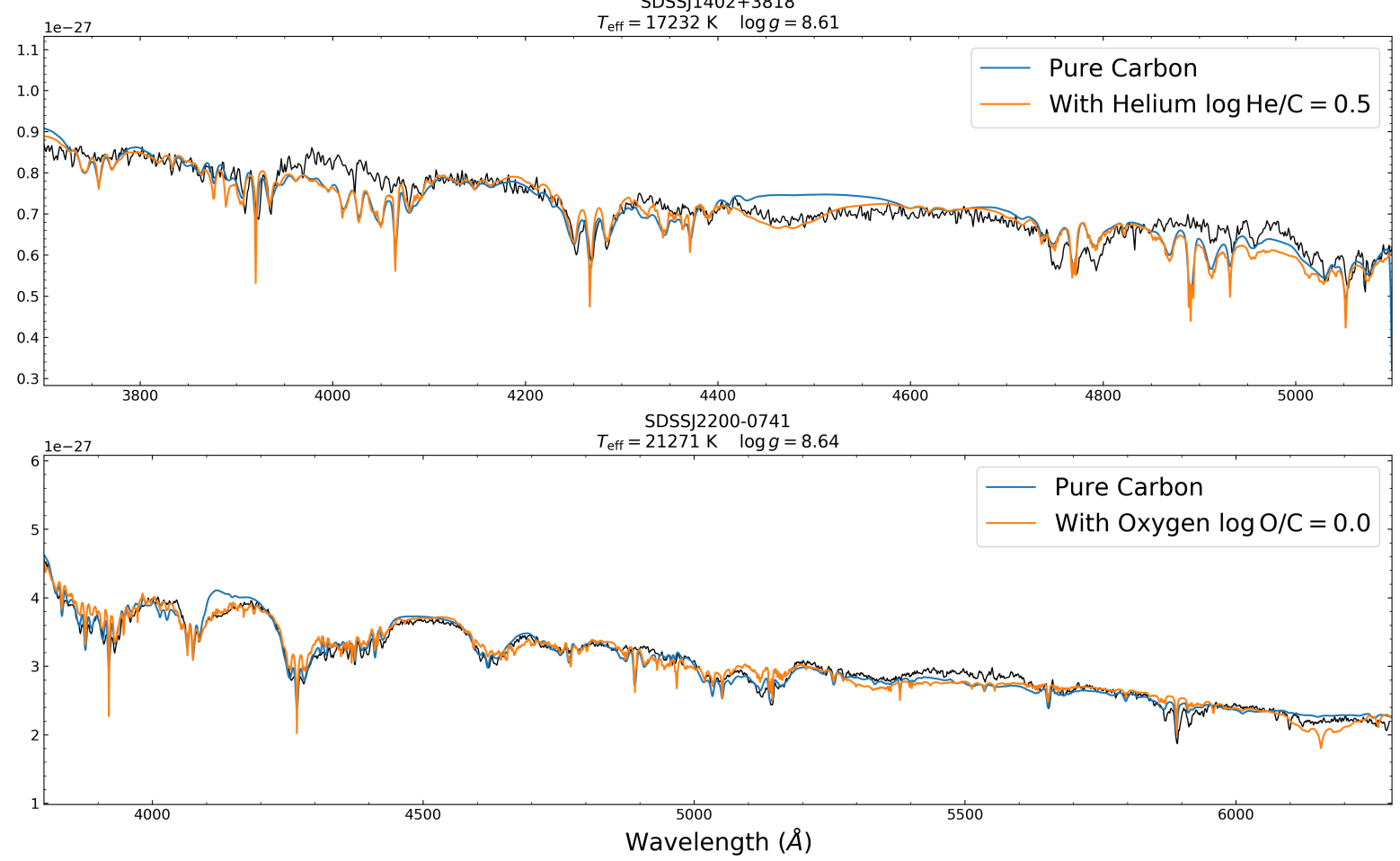

Figure 3: Pure carbon atmospheres (blue) for 4 Hot DQ stars do not fit the spectrum perfectly, as some features are best fitted with the addition of other elements such as hydrogen, helium, and oxygen. For J1337, two elements other than carbon were detected: hydrogen (red ticks) and oxygen (green tick). The features for the other stars are self-explanatory. 